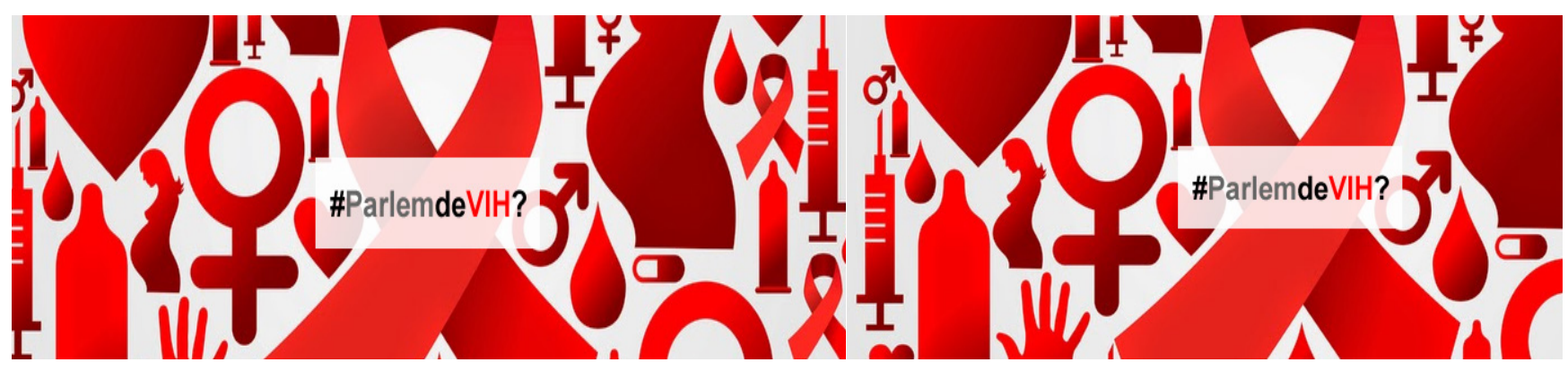

\title{
III Jornadas Internacionales Comunicación y Salud: \#ParlemdeVIH III International Conference Communication and Health: \#ParlemdeVIH
}

$\square$ n el ya clásico Le sida, Jerome Strazzula (1993: 15) escribe que "la epidemia representa algo más que una enfermedad, el sida es un fenómeno mediático: los medios «revelaron» al mundo la existencia de esta patología y también los medios han sido decisivos en la propia historia de la enfermedad". 25 años más tarde dedicamos las III Jornadas de Comunicación y Salud a reflexionar en torno a la comunicación y el $\mathrm{VIH}$.

A finales del 2005, el Observatorio de Derechos Humanos de Red2002 emprendió el proyecto "Una nueva imagen para el VIH", que surgió de la necesidad de mejorar la manera en que se ofrecía la información relacionada con el $\mathrm{VIH} /$ sida en los medios de comunicación en España; el ODH encarga al Observatorio de Comunicación y Salud -OCS- (InCom-UAB) un estudio sobre el tratamiento del $\mathrm{VIH} /$ sida en los medios de comunicación escritos españoles con la finalidad de elaborar una guía de buenas prácticas dirigida a los periodistas encargados de redactar piezas sobre el $\mathrm{VIH} /$ sida y, de esta forma, contribuir a propiciar un cambio en la imagen que ofrecen los medios sobre el VIH/sida. Este proyecto inicial varió sustancialmente (Terrón, 2013). Por un lado, se alargó en el tiempo (entre 2006 y 2010) y, por otro, dio pie a una serie de acciones cuyo objetivo fue buscar una mayor complicidad entre los medios de comunicación y las ONG que trabajan en el ámbito del VIH y, así, afianzar la cooperación. Fruto de la experiencia adquirida, el OCS replicó la investigación y sus propósitos en México (Sánchez Maldonado, M.; Terrón, J.L.; Lozano Rendón, J.C, 2016) y República Dominicana (Terrón, 2015).

Tal como apuntó Alejandro Brito (2007:13), "comunicadores e informadores ya no perciben al $\mathrm{VIH} /$ sida como noticia, "no estamos ante un tema novedoso", arguyen. Aparentemente, sobre esa epidemia ya se dijo todo lo que se tenía que decir". En 2013 apuntábamos que "sólo la construcción de relatos con nuevos enfoques y nuevos datos posibilitaría la permanencia en la agenda de los medios, dado que la noticia que esperan todas las redacciones es la consecución de una vacuna; de ahí que cualquier indicio en este sentido se torne en noticia destacada" (Terrón, 2013: 59); parecía que en Occidente, siguiendo a M. Nicolás y M. Martín, el VIH/sida sería un 'postproblema'. En 2018, ¿podemos mantener este punto de vista? Sospechamos que no, de ahí la necesidad de relanzar las reflexiones en torno a las relaciones que pueden establecerse entre mediaciones comunicativas y VIH. De ahí la oportunidad de organizar unas jornadas que sirvan como marco de esas reflexiones y como punto de inflexión en nuestro país para, de nuevo, pensar en el rol de la comunicación, de sus mediaciones, cuando hablamos o tratamos del VIH o del sida.

Las III Jornadas de Comunicación y Salud: \#Parlemde$\mathrm{VIH}^{1}$ se desarrollarán en Barcelona los días 21 y 22 de noviembre de 2018 en la sede del Institut d'Estudis Catalans y están organizadas por el SCOMLab (Laboratoria de Comunicación y Salud del InCom-UAB). La inscripción es gratuita, y para la realización de las Jornadas coloboran, entre otros, la Societat Catalana de Comunicació, la Revista Española de Comunicación en Salud, La Generalitat de Catalunya o el Ayuntamiento de Barcelona.

El 21 de noviembre se dedicará a la presentación online de comunicaciones, para lo cual se contará con un canal específico de Youtube. Las propuestas podrán presentarse hasta el 20 de septiembre de 2018; todos los resúmenes aceptados se publicarán en formato digital (acceso abierto). Por otro lado, los autores podrán enviar los textos completos para ser publicados en formato de

$1 \mathrm{http}: / /$ comunicacioisalut.cat/es/; también se puede seguir la actualidad sobre las mismas a través de Twitter: @parlemdevih 
artículo científico en la Revista Española de Comunicación en Salud (RECS), tras haber superado el proceso de evaluación establecido por la publicación. El canal Youtube permanecerá activo más allá de las Jornadas y en él se incorporarán las grabaciones de las mesas que se llevarán a cabo el día 22.

Las propuestas enviadas han de tratar sobre alguno (0 algunos) de estos temas:

- Salud Pública

- Promoción de la salud y prevención de enfermedades nicación

- Periodismo y salud en los medios de comu-

- Campañas de salud

- Comunicación con el usuario-paciente

- Sociedad-salud-bienestar

- Publicidad y salud

- Emergencias y crisis en salud

- Tele-salud, tele-medicina, eHealth y Salud 2.0

- Terapias digitales

- Cine y salud

- Educación y salud

- Investigación en comunicación y salud

Durante el día 22 se desarrollará la sesión presencial, que será retransmitida vía internet. La misma se iniciará con una conferencia en la que se reflexionará sobre el $\mathrm{VIH}$ desde una perspectiva de género, a la que le seguirán cuatro mesas redondas:

Prácticas, para dialogar sobre prácticas de riesgo o de prevención que no suelen tener un espacio relevante en los medios de comunicación (por ejemplo, el chemsex o el PReP).

APP, para analizarlas en relación al VIH y el sida desde distintas perspectivas: por un lado, como herramientas médica, de empoderamiento o solidaria, pero, por otro, como útil que conforman estilos de vida que pueden repercutir en las prácticas de riesgo, en la privacidad, en la estigmatización y en la prevención.

.Comunicación, y, así, reflexionar sobre cómo tratan los medios de comunicación el $\mathrm{VIH} /$ sida, a la vez que evaluar las campañas de prevención.

.También cuentan, con el propósito de dar visibilidad a aquellas personas o colectivos que viven con VIH y de los que no se suelen hablar en los medios de comunicación (mujeres, mayores, latinos, presos,...).

En las cuatro mesas se enfocarán los distintos temas desde las perspectivas de las personas que viven con $\mathrm{VIH}$, los periodistas y los profesionales de la salud. Tras su finalización, y en colaboración con el Barcelona Inter- national LGTIB Film Festival, se proyectarán algunas de las películas de su última edición que tengan como uno de sus argumentos el VIH. Por otro lado, y de manera permanente, el SCOMLab administrará un canal en Filmin que se dedicará en exclusiva a relatos audiovisuales (documentales o de ficción) en los que se trate del VIH o del sida.

¿Por qué volver a hablar del VIH? ¿Por qué hacerlo desde la perspectiva en la que se enmarcan las Jornadas? Nos gustaría ofrecerles algunos datos, la mayoría de ellos extraídos del informe Vigilancia epidemiológica del VIH y sida en España 2016² (actualizado a 30 de junio de 2017) que elabora el Instituto de Salud Carlos III.

En España, con datos de 2016, hay alrededor de 145.000 personas que viven con $\mathrm{VIH}$-de las cuales, unas 32.000 son mujeres-; aproximadamente una de cada cinco personas (18\%) no está diagnosticadas. El $46 \%$ de los nuevos diagnósticos se realizó de forma tardía y la tasa de nuevos diagnósticos de VIH es superior a la media de la UE y de los países de Europa Occidental. Alrededor del $26 \%$ de los infectados tenía menos de 30 años cuando fue diagnosticado y un $16,4 \%$ tenía 50 años o más; y el $33,6 \%$ de los nuevos diagnósticos fue en personas nacidas fuera de España: tras el origen español, el más frecuente fue el latinoamericano (16,6\%). De las personas que tienen sida, el porcentaje de las que se infectaron por relaciones heterosexuales asciende al $36,2 \%$ y el $32 \%$ en hombres que tienen sexo con hombres. Según el Observatorio del Sistema Penal y Derechos Humanos de la Universidad de Barcelona, el riesgo de tener VIH dentro de una prisión catalana es entre 20 y 28 veces más elevado que fuera. Mientras que en Catalunya el porcentaje de personas que tienen $\mathrm{VIH}$ es del $0,27 \%$ este porcentaje aumenta hasta el 7,6\% en el caso de las personas presas. Las mujeres presas sufren un índice de prevalencia del VIH más alto $(11,6 \%)$ que los hombres $(8,85 \%)$. Mientras que la prevalencia del VIH en el total de la población es del 0,4, entre las trabajadoras del sexo asciende al $2 \%$, entre los hombres que tienen sexo con hombres al $11,3 \%$, un $2,3 \%$ entre los que se inyectan drogas, el $5,4 \%$ entre los presos y un $11,3 \%$ entre las mujeres trans.

¿Los medios han dejado de hablar del VIH y del sida? Esa es la percepción mayoritaria; por nuestra parte parte (Terrón, J.L; Ramírez, F.; Vialás, S.; Jacobetty, P., 2017) podemos apuntar que algunos diarios -El País y El Mundo- no lo han dejado de hacer. Eso sí, esos mismo diarios entre junio de 2016 a junio de 2017 dedicaron 22 piezas

\footnotetext{
${ }^{2}$ https://www.msssi.gob.es/ciudadanos/enfLesiones/enfTransmisibles/sida/ vigilancia/InformeVIH_SIDA_2017_NOV2017.pdf
} 
(sobre 697) a tratar sobre el PReP (12 El País y 6 El Mundo) y 3 a escribir sobre el PEP (2 El País y 1 El Mundo). Lo que nos da pie a que nos preguntemos, una vez más, si los relatos y los enfoques son los adecuados; quizás debemos olvidarnos un tanto de cuánto se publica y poner nuestra atención en qué y cómo se publica. Siguiendo con el ejemplo que acabamos de exponer, ¿cómo es posible que los medios lo traten de manera tan escasa un tema como el del PReP a pesar de las recomendaciones de la propia OMS para su uso y de que España siga sin implantarlo? ¿Han visto alguna campaña de alguna administración o institución a favor del PReP?

Ejemplos como el anterior, nos muestran que sigue siendo deseable y necesario pensar en el $\mathrm{VIH}$ desde la comunicación, que no todo está dicho, ni que todo está bien contando; y que siempre faltan voces que deben de ser escuchadas. De ahí que nos parezcan oportunas unas Jornadas como estas, a las que están todos vds. invitados.

\section{Referencias Bibliográficas}

Brito Lemus, A. (2007). Sida, estigma y discriminación. Guía práctica de manejos de medios, México: Letra $S$, Salud, Sexualidad, Sida; suplemento de La Jornada.

Sánchez Maldonado, M., Terrón, J.L., \& Lozano Rendón, J.C. (2016). Estigmatización y usos léxicos $n$ el tratamiento informativo del $\mathrm{VIH} /$ sida en cinco diarios mexicanos de 2013 a 2013, Comunicación y Sociedad, 25, 71-100.

Strazulla, J. (1993). Le Sida, París: La documentation Française.

Terrón, J.L., Ramírez, F., Vialás, S., \& Jacobetty, P. (2017). Cómo informan El País y El Mundo sobre la estrategia preventiva PrEP contra las infecciones del $\mathrm{VIH}$, 116-117, en VV.AA. El fin de un modelo de política. La Laguna: Cuadernos Artesanos de Comunicación.

Terrón, J.L. (2015). El tratamiento del VIH/sida en los medios impresos de España y de la República Dominicana: el papel de las ONG en la formación de la opnión pública, 209-226, en Reñó, D., Martínez, M., \& Campalans, C. (coord.). Medios y opinión pública, Bogotá: Editorial Universidad del Rosario.

Terrón, J.L. (2013). El tratamiento del VIH/sida en los pe riódicos españoles, investigar para propiciar nuevos relatos periodísticos, Comunicação e Sociedade, 23, 57-68.

\section{José Luis Terrón Blanco}

Comité organizador 